\title{
La risa en la poesía de Francisco Álvarez de Velasco y Zorrilla*
}

Fecha de recepción: 15 de junio de 2017

Fecha de aprobación: 11 de septiembre de 2017

\section{Resumen}

La Rhythmica sacra, moral y laudatoria (1703), de Álvarez de Velasco y Zorrilla, se revela como un testimonio, único en la Nueva Granada, de las distintas manifestaciones de la risa en la poesía colonial. Primero, evidenciamos la exclusión de la risa en la "ciudad letrada", y la consecuente escritura de sátiras no burlescas. Como objetivo lateral, en estas piezas analizamos también la apropiación criolla de los modelos peninsulares. Segundo, estudiamos los poemas asociados a fiestas religiosas por la pervivencia de la risa popular. Tercero, localizamos una esfera intermedia en las cartas laudatorias a Sor Juana, donde la risa se independiza de la fiesta y emprende el camino a la modernidad.

Palabras clave: Estética de la risa; Álvarez de Velasco y Zorrilla; poesía colombiana; literatura colonial; sátira; burlesco.

\section{Guillermo Molina Morales}

Profesor e investigador del Instituto Caro y Cuervo. Candidato a Doctor en Teoría de la Literatura de la Universidad de Zaragoza.

Guillermo.Molina. Morales198@gmail.com

* Artículo de investigación enmarcado en el proyecto de investigación "La risa en la poesía hispana de la Nueva Granada"

Citar: Molina Morales, G. (julio-diciembre de 2017). La risa en la poesía de Francisco Álvarez de Velasco y Zorrilla. La Palabra, (31), 119-130. doi: https://doi. org/10.19053/01218530.n31.2017.7282. 


\section{Laughter in the Poetry of Francisco Álvarez de Velasco y Zorrilla}

\section{Abstract}

Rhythmica sacra, moral y laudatoria [Sacred, moral and laudatory rhythmics] (1703), by Álvarez de Velasco y Zorrilla, is a unique Neogranadian document that studies the diverse expressions of laughter in Colonial poetry. Firstly, we show the exclusion of laughter in the "high culture", and the consequent writing of non-burlesque satires. As a side objective, in these poems we also analyze the creole appropriation of Peninsular models. Secondly, we study the works associated with religious rituals and the persistence of laughter in the common people. Thirdly, we propose an intermediate field in the laudatory letters to Sor Juana, where laughter becomes independent from these rituals and initiates the way to modernity.

Key words: aesthetics of laughter; Álvarez de Velasco y Zorrilla; Colombian poetry; Colonial literature; satire; burlesque.

\section{Le rire dans la poésie de Franciso Álvarez de Velasco y Zorilla}

\section{Résumé}

La Rhythmica sacra, moral y laudatoria [La Rhythmica sacrée, morale et laudative] (1703), de Álvarez de Velasco y Zorrilla serait un témoignage, exceptionnel, dans la Nouvelle-Grenade, des différentes manifestations du rire dans la poésie coloniale. D'abord, nous mettons en évidence de quelle manière le rire est exclu de "la ville lettrée", et par conséquent se met en place l'écriture de satires non burlesques. Nous analyserons l'appropriation locale des modèles péninsulaires dans ses textes. Dans une deuxième partie, nous étudierons les poèmes associés aux fêtes religieuses dans lesquels le rire populaire est maintenu. Dans une troisième partie, nous nous demanderons dans quelle mesure dans les lettres laudatives à Sor Juana, le rire s'émancipe de la fête et entreprend un chemin vers la modernité.

Mots-clés: esthétique du rire, Álvarez de Velasco y Zorrilla, poésie colombienne, littérature coloniale, satire, burlesque. 


\section{Introducción}

En enero de 1771, es ofertada en oposición pública la encomienda de Serinza, perteneciente al Nuevo Reino de Granada, en el actual departamento de Boyacá (Colombia). Uno de los candidatos arguye en la postulación su origen noble $y$, particularmente, el hecho de ser descendiente de insignes caballeros que lograron cortar y estacar cabezas de numerosos indios sin costo alguno para la Real Hacienda (Porras Collantes, 2000, p. 15). Quien esto suscribe, Francisco Álvarez de Velasco y Zorrilla (Santafé de Bogotá, 1647; Madrid, 1708), publicaría más tarde un libro titulado Rhythmica sacra, moral y laudatoria, en el que se precia de excluir la risa, por ser asunto viciado y más propio de barberos (p. 5). A cambio, el autor afirma ofrecer "verdadera, decente y rigurosa poesía" (p. 7), en consonancia con la castidad y pureza moral que predica.

La publicación de este libro en España, en el año 1703, permitió salvar del olvido a este destacado representante de la "ciudad letrada". De hecho, Álvarez de Velasco y Zorrilla es considerado, hoy en día, como unos de los principales poetas de la Nueva Granada colonial, lo que se explica, entre otras cosas, por no haberse conservado otras colecciones de poemas de similar tamaño (recordemos que la imprenta no llega a la ac- tual Colombia hasta bien entrado el siglo XVIII). La afición de escribir versos era muy común en la élite criolla de su tiempo, sobre todo, con motivo de celebraciones civiles y religiosas, pero pocas veces se intentó (o se logró) la publicación de estas obras.

En este contexto, el estudio de la Rhythmica sacra, moral y laudatoria, reeditada por el Instituto Caro y Cuervo en el año 1989, resulta interesante por revelarnos las concepciones y prácticas literarias habituales en la élite santafereña de finales del siglo XVII. Se trata de un periodo en el que dominaba el estilo barroco, del cual, según Torres Quintero (1989), nuestro autor es una "especie de enciclopedia" (p. XVI). Este hecho generó que la crítica tradicional (desde sus comienzos, a mitad del siglo XIX, hasta mediados del siglo $\mathrm{XX}$ ) considerara de forma despectiva la obra de Álvarez de Velasco. Curiosamente, el propio poeta, apoyándose en su admiración hacia Quevedo, declaraba la aversión hacia Góngora y sus seguidores, a quienes llama "songoreadores de las Musas" y "Gimios del Parnaso" (p. 7). Sin embargo, este rechazo teórico no le impidió practicar, en la mayor parte de los poemas conservados, un estilo notablemente artificioso.

De esta manera, se entiende el juicio del clasicista Menéndez Pelayo (1952), quien considera- ba a nuestro autor, al igual que a Domínguez Camargo, como un "ingenio malogrado por la educación y el medio" (p. 425). De forma análoga, Gómez Restrepo (1953) denuncia que el poeta "tenga frecuentes caídas de gusto e incurra en ocasiones en lo chabacano, defecto muy común en la poesía de entonces" (p. 165). Por su parte, Otero Muñoz (1945) habla de "composiciones tan laboriosas como ininteligibles" que reflejan "resabios de mal gusto llevados a la exageración" (p. 36). Entre los críticos tradicionales, solamente Arango Ferrer (1965) valora positivamente su ingenio verbal, llegándole a considerar como un antecedente de las greguerías (p. 107).

En las últimas décadas, la obra de Álvarez de Velasco ha disfrutado de una tímida revaloración. La "Carta laudatoria" a Sor Juana, junto con otros poemas dedicados a la poeta mexicana, ha despertado un especial interés (interés que, por cierto, tiene un lejano precedente en el Papel periódico de Manuel Socorro Rodríguez, a finales del siglo XVIII). Así, Pascual Buxó (1999) destaca la forma en que "el poeta colombiano enamorado de sor Juana" muestra su admiración siguiendo la retórica del neoplatonismo y del "amor cortés". Por su parte, Jurado Valencia (2000) estudia la obra de Álvarez de Velasco como ejemplo de recepción, e incluso de 
explicación y legitimación, de la poesía de Sor Juana.

También, se muestra complaciente con nuestro autor el crítico Héctor Orjuela (1992), quien lo califica como "el poeta innovador por excelencia en nuestras letras coloniales" (p. 28), si bien ese "gusto por la novedad" se limita a la experimentación métrica y rítmica, y a los juegos de ingenio. En todo caso, Orjuela parece preferir una sencilla composición amorosa, "Buelve a su quinta Anfriso solo, y viudo", que él considera "la máxima expresión en su obra lírica" (p. 43), y que relaciona con la poesía neoclásica posterior. De forma análoga, Bechara (1997) estima el posicionamiento de Álvarez de Velasco a contracorriente de las corrientes gongorinas: "es como si Álvarez de Velasco reclamara a gritos el retorno al clasicismo, a la elegancia sencilla" (p. 275). Por otro lado, reconoce que el poeta cultivó con asiduidad la "poesía artificiosa", de cuyas piezas más complejas realiza un detallado análisis formalista.

A diferencia de los estudios anteriores, la presente investigación pretende indagar en las concepciones y prácticas que muestra la poesía de Álvarez de Velasco y Zorrilla en torno a la risa. Este enfoque puede parecer sorprendente, por cuanto el propio autor explicita la exclusión de poesías "profanas y jocosas" (p. 5), que considera "sobre pueriles, delincuentes" (p. 6); y, en cambio, manifiesta optar por "la legitima y verdadera Poesía", que se fundamenta en "la nobleza de los objetos" (p. 7). Este énfasis del poeta en el "Prólogo al lector" es, desde luego, interesante para el estudioso de la historia de la risa. Sin embargo, más allá de la declaración de intenciones, interesa analizar los poemas en sí mismos. En ellos, encontramos tres tipos de prácticas muy significativas: la sátira seria (en la parte "moral" de la Rhythmica), la pervivencia de la risa popular (en la sección "sacra") y una "esfera intermedia" (en el capítulo "laudatorio" dedicado a Sor Juana). Para su análisis crítico, nos basaremos en la Estética de la risa del profesor Beltrán Almería (2016), cuya perspectiva amplia aplicamos, por primera vez, al contexto colonial.

\section{Rhythmica moral, o la sátira criolla sin risa}

En las últimas décadas, autores como Mabel Moraña han destacado la originalidad de las manifestaciones del barroco en Hispanoamérica, que ya no se pueden entender de forma automática como mera copia o reflejo de la literatura peninsular. Con todo, el punto de partida para estudiar las "apropiaciones" de los criollos, en especial del sector letrado, siguen siendo los "códigos metropolitanos hegemónicos" (1989, p. 227). Algunos de estos códigos funcio- nan, al menos en la teoría, como axiomas centrales que articulan las producciones coloniales, incluso más allá del periodo de dominación española. Uno de estos códigos, acaso el más profundo, es la separación entre la seriedad y la risa.

Beltrán Almería (2016) afirma que "los humanistas eran elitistas y defendían la pureza de la seriedad, en la vana esperanza de que la dignificación de lo serio sirviera para alejar a la humanidad de sus registros animales y bárbaros" (p. 12). Este empeño debía ser particularmente intenso en una sociedad que, precisamente, estaba superpuesta a unos pueblos indígenas considerados como incivilizados. El propio Álvarez de Velasco exalta la nobleza del hombre racional, que "lo haze participante de la naturaleza Angelica", y advierte que "quanto mas exercita lo animal, pierde lo racional" (p. 602). Las pasiones, entre las que se incluye de forma destacada la risa, deben, por lo tanto, excluirse de la "ciudad letrada", lo que justifica el rechazo de la poesía "jocosa" que hemos visto más arriba.

Por este motivo, no debe extrañarnos que en la poesía satírica de Álvarez de Velasco se produzca una marginación del delectare a favor del puro docere. Para entenderlo, nos parece útil la distinción que establece Arellano (2006) entre la sátira, 
definida por su intención moral, y lo burlesco, término que estaría ligado al estilo jocoso. De sus posibles cruces, surgen tres modalidades: poemas satíricos no burlescos, poemas satíricos burlescos y poemas burlescos no satíricos.

Esta aclaración cobra especial relevancia en el caso del poeta santafereño. Orjuela (1992), mostrando una buena intuición pero escasa claridad conceptual, había señalado que "la poesía satírica de Álvarez de Velasco y Zorrilla tiene tono moralizante y deja traslucir cierta intención burlesca, pero carece de humor y de cierta picardía que a veces se encuentra en este tipo de composiciones" (p. 40). Esta poesía satírica moralizante y carente de humor, se puede entender como sátira no burlesca, lo que concordaría con el carácter ascético y estoico, de meditación moral, que el propio Orjuela localiza en el autor (p. 36). Veamos un ejemplo en la estrofa final del poema "Lo que passa en los aplausos del mundo", a la que precede la muy esquemática anécdota en la que el sujeto poético se encuentra en la calle a gente que aplaude a un loco:

Con que pude sacar este discurso:

Luego del vulgo el sequito, y concurso

Se va a los locos, y a los sabios dexa? [...]
Solo me quiero andar, no acompañado;

De buena gana esse bullicio pierdo (p. 302).

Encontramos aquí la desnuda enunciación de una verdad moralista: la superioridad del sabio solitario frente al bullicio popular. La crítica, en este caso, no se sirve de la risa como herramienta de ridiculización. $\mathrm{Ni}$ siquiera la inclusión de un loco en la escena propicia algún detalle de jocosidad en el poema, lo que puede compararse con el protagonismo de la risa del loco como medio de crítica social en algunos poetas contemporáneos (Gaitán Bayona, 2015).

Por supuesto, ni la moraleja ni la supuesta anécdota narrada en primera persona son originales de Álvarez de Velasco (existe una larga tradición cuyo origen se atribuye al político griego Foción, según lo cuenta Plutarco en sus Vidas paralelas). Recordemos que, al menos hasta el Romanticismo, la literatura se concebía como recreación de modelos reconocidos. Además, debemos añadir que el aspecto esencial de la sátira, según Beltrán Almería (2002), es la "tipificación", entendida como opuesta de la "caracterización", o construcción de un carácter (p. 259). Así, el "tipo" sería "un personaje que prescinde por completo de su alma, de su individualidad" (p. 260). Algunos de los tipos que caricaturiza Álvarez de Velasco son el soberbio, el pedante y el avaro, todos ellos presentes en múltiples culturas. Por este motivo, resulta muy difícil la tarea, que suelen asumir los estudios coloniales en las últimas décadas, de explicitar la peculiaridad de la expresión americana.

Una solución, adoptada por Jaime Tello (1989) en las notas de la edición moderna, es señalar hacia quién va dirigida la sátira, utilizando para ello informaciones extraliterarias. De esta manera, por ejemplo, establece que el "Hipocóndrico ocupado, como todos los de su profession, en continuas curas" es "sin duda, don Francisco del Castillo de la Concha, Presidente-Gobernador del Nuevo Reino de 1679 a 1685 , contra quien Álvarez de Velasco se pronuncia varias veces de manera violenta", y que de hecho había sido caracterizado como hipocondríaco en una crónica (p. 300). Carecemos, sin embargo, de más informaciones que permitan estudiar si este tipo de ataques satíricos tuvieron alguna repercusión social, como solía ser común tras la llegada de la imprenta en pasquines o publicaciones periódicas.

Otra vía de estudio podría ser la frecuencia con que se prefieren ciertos temas tradicionales sobre otros. En este sentido, detectamos una notable insistencia en las sátiras contra jueces y escribanos, en poemas como "Sermón eneuclástico" y “A 
Pilatos, y en él a los juezes". También en el "Novissimo del infierno" (versión del Inferno dantesco) encontramos de forma destacada a funcionarios como "Letrados (Linces ciegos)", "mañosos palaciegos", "ministros placenteros", etc. (p. 176). Y, lo que es más relevante, en "Definición de la vida" se habla de "algun villano Juez de Residencia / O de algun mal Oidor, o Presidente, / Preciados en lo terco de inhumanos" ( $\mathrm{p}$. 217). Es importante destacar que las figuras del juez de residencia y de oidor fueron prácticamente exclusivas de América, al menos desde el siglo XVI. En cuanto al "Presidente", por el contexto podemos pensar que se refiere al Presidente de la Audiencia de Santafé de Bogotá.

Sin ser tan obsesivo como su contemporáneo Juan del Valle y Caviedes respecto a los médicos, Álvarez de Velasco muestra una inclinación hacia la sátira de la burocracia colonial, pero siempre en el contexto del moralismo cristiano, por lo que el castigo se pospone a la vida ultraterrena. Así, las ya citadas figuras del Juez de Residencia y el Oidor aparecen en un poema dedicado al tópico del desengaño del mundo, es decir, de las vanas pompas de la vida sensible, calificada esta última como "Perspectiva de sombras colorida, / Venenosa hechizera musaraña" (p. 216).
Entre los poemas satíricos, uno ha llamado especialmente la atención de la crítica: el soneto dedicado "A un linajudo mordaz, y sin mucha razon presumido de Nobleza muy antigua". De este poema, escribió Gómez Restrepo (1953) que "revela en su autor cierto sentimiento democrático" (p. 167), mientras que Orjuela (1992) lo asocia a "la poesía de crítica social tan abundante en algunas generaciones posteriores" (p. 40). Nuestra postura se acerca más a la de Bechara (1997), quien contradice a Gómez Restrepo por considerar que el poeta se muestra en todo punto coincidente con la ideología imperial y, más bien, explica este poema por imitación a Quevedo (pp. 267-268). Una lectura atenta nos advierte de que la sátira es, en realidad, de carácter moral, y no social o político. Tras el terceto en que aconseja "No, pues, para infamar Generaciones / En trasegar sepulcros busques peste, / Ni en los de tus abuelos más blasones", concluye con "Sea la humildad el tuyo" (p. 424). Se trata, como vimos en los poemas anteriores, de recomendar la moderación frente a las apariencias terrenales, con la vista puesta en el plano celeste, y no tanto en los problemas terrenales.

Por este motivo, y por otros que enseguida veremos, nos parece demasiado aventurado interpretar en Álvarez de Velasco algún tipo de deseo de diferenciación respecto a la metrópoli, a pesar de sus referencias a la Nueva Granada y de la inclusión de algunos americanismos. Respecto al contexto neogranadino, confirmamos las ideas apuntadas con poemas como "A Lelio", que explícitamente se dedica a "un Governador", pero está compuesto por "solos principios de distintos Sonetos de Quevedo" (p. 308). Es decir, aquí se produce un trasplante literal de versos peninsulares para referirse a circunstancias locales (circunstancias que, por otro lado, tampoco son el objetivo principal de esta sátira moral).

En cuanto al uso de americanismos, autores como Tello (1989) lo han interpretado como una demostración del "legítimo orgullo de ser americano" (p. XXXIX). Es indudable el hecho de que se incorporan algunas palabras y expresiones locales, pero hay que ser cuidadoso a la hora de atribuir a los escritores coloniales sentimientos que, tal vez, solo formen parte del crítico contemporáneo. De hecho, en la "Advertencia y protesta" con que termina el libro, Álvarez de Velasco justifica el empleo de estos términos por creer "que eran corrientes en todo España", y advierte que estos "defectos" los hubiera enmendado "a no avermelos advertidos despues de estar ya todos impressos" (p. 620). No sabemos el grado de sinceridad o de estrategia de esta declaración, escrita en un libro publica- 
do en España, pero en todo caso queda lejos de ser susceptible de una interpretación en clave proto-nacional.

Con lo dicho anteriormente, podemos extraer algunas conclusiones sobre el criollismo de Álvarez de Velasco. Para ello, es necesario abandonar el providencialismo nacionalista de sus críticos; por ejemplo, el de Orjuela (1992) cuando declara que el poeta "anuncia una nueva época" y que con él "surge el verdadero poeta criollo que quiere cantar a América con un idioma americano" (p. 50). El propio sintagma de "verdadero poeta criollo" es excesivamente problemático si pensamos, como Mazzotti (1996), que no se puede dar una definición monolítica de la identidad criolla. En este caso concreto, el criollismo se manifiesta como una continuación de los modelos peninsulares, particularmente de Quevedo, llevados, en algunas ocasiones, al marco referencial de la Nueva Granada. Si hay un deseo de diferenciación, es respecto a los indígenas, lo que era muy común en la ciudad letrada (Mazzotti, 1996, pp. 173-175). Esto último se ve claramente en sonetos como el dedicado "A la milagrosa echura del santo Christo de Maracaybo", estatua cristiana considerada como vivo testimonio de la maldad de los "Indios Gentiles" (p. 417).

La postura de Álvarez de Velasco recuerda la oposición entre "criollismo" y "mestizaje" que establece Rafael Bernal (2015) para la literatura novohispana. En este sentido, la poesía del santafereño se puede definir como "una literatura criolla, esto es, un trasplante del mundo estrecho de España y el ecúmeno mediterráneo a la fecundidad y riqueza de América" (p. 20). La diferencia más profunda en este proceso de trasplante, creemos, sería la exclusión de la risa en la sátira, que no podemos atribuir con seguridad a una causa individual o social. En cualquier caso, queda claro que, en estos poemas, el autor opta por un moralismo estricto que no hace concesiones al delectare, lo que resulta especialmente relevante por cuanto se aparta de su reconocido modelo peninsular, Francisco de Quevedo.

Rhythmica sacra, o la pervivencia de la risa popular

Después de lo analizado en el epígrafe anterior, puede resultar muy chocante encontrar un poema, "Al nacimiento de Christo", que comienza de esta manera:

"Adivina, adivinador, Qué cosa tan nueva, y de tanto primor, Que siendo un ogar pajizo Está buelto un Paraiso, Que sirve de Alcaçar Real, Y yo lo juzgo Por-tal?"'(p. 356).

A primera vista, dos aspectos llaman la atención: en primer lugar, el empleo de una estructura de juego infantil popular que todavía hoy persiste entre los niños bogotanos; en segundo lugar, el calambur de la última palabra, entre el Portal de Belén y la expresión "por tal". Por otro lado, aquí hay una ausencia de crítica y de tono moralizante. Nos encontramos, por lo tanto, en el otro extremo del espectro que propone Arellano (2006), es decir, en la poesía burlesca no satírica, "atenta únicamente al delectare y a la diversión risible que procede del alarde estilístico" (pp. 340-341). Otros versos del mismo poema confirman la intuición, como la siguiente secuencia pregunta-respuesta: "Y esse Niño es muy chiquito? / Ya tiene sus Navidades" (p. 343), en la que de nuevo se juega con el doble significado de las palabras en una expresión, por cierto, de origen popular.

Esta aparente contradicción entre la exclusión de la risa en la poesía moral y el juego festivo con las palabras en algunos poemas de contenido sacro, se explica si seguimos el razonamiento de Beltrán Almería (2016). Según este teórico, el predominio de una alta cultura seria, característica de la "ciudad letrada", relegó el mundo de la risa al ámbito de la cultura popular (p. 17). Esta cultura popular se manifiesta con preferencia en el tiempo de la fiesta y se expresa, entre otros recursos, a través de juegos verbales (p. 23). No queremos decir con 
esto que Álvarez de Velasco sea un poeta popular (de hecho, lo popular se sustenta en la oralidad, y no en la escritura), pero sí que se acoge a los códigos estéticos de este ámbito en la composición de varios poemas.

En casi todas las ocasiones, se trata de poemas compuestos con motivo de una festividad religiosa, como el villancico navideño cuyo comienzo hemos copiado más arriba. Es de suponer, aunque no lo sabemos realmente, que estas piezas estaban destinadas para el canto, o al menos para la recitación, en las fechas señaladas. Las formas métricas y estróficas se corresponden con las habituales en la poesía española de carácter popular. De esta manera, es común el verso octosílabo formando cuartetas y quintillas, en contraposición al endecasílabo, verso de arte mayor que se consideraba más apropiado para los poemas serios. También es característica la inclusión de expresiones coloquiales y de juego con el lenguaje, como en los versos "no se hallava un pobre ciego / por un ojo de la cara" (p. 374), pertenecientes a la "Vida, $\mathrm{y}$ charidad del gran patriarca de Alexandría San Juan Limosnero".

Este último poema, por cierto, puede parecer sorprendente desde una perspectiva actual: se cuenta aquí la vida de un hombre tan generoso que, finalmente, Dios "por dexarnos algun pobre, / trató de llevarse al Santo" (p. 376). En un momento anterior del poema, el Creador, al ver casado al protagonista, "por darle las dichas llenas, / la muger le llevó apenas" (p. 373). Una lógica, como vemos, más cercana a la cultura popular que a la ortodoxia católica (hoy diríamos que, como mínimo, no es "políticamente correcto" propiciar la muerte de alguien por su mera condición de esposa). Algo similar encontramos en la "Vida, y milagros del nuevo taumaturgo, y apostol de las Indias S. Francisco Xavier", quien entre otras "trabesuras" practicaba la resucitación, y "tenia amistad con los Curas / con [a pesar de] quitarles los entierros" (p. 368). Obviamente, desde una perspectiva seria, un santo no compite con los sacerdotes por resucitar o dejar morir a una persona.

Interesa también abordar "Otro villancico al mismo Nacimiento de Christo S. Nuestro", ya que presenta un caso de poliglosia paródica entre el latín y el castellano. El poema simula una discusión escolástica, pero no pretende demostrar una idea (de hecho, no se llega a ningún acuerdo), sino jugar libremente con las palabras de forma burlesca. Copiemos un pequeño fragmento, en torno a la disputa "sobre si el Niño, / que oy nos nace es Nombre, o Verbo":

1. Ea, pues, si es nombre por donde se declina? diga presto.

2. Por sensus, sensus. 1. Jesus!

Verbum caro! Qué soleco!

2. Pues Jesus por donde va?

1. Por Dominus. 2. Bueno, bueno,

appinge, appinge duo in illum,

gané el vando sin remedio (pp. 349-350).

Como se ve, el contenido religioso o didáctico queda en un segundo plano, y pasa al primero el disfrute de las palabras, en una mezcla de latín, español académico ("se declina"), español académico vulgarizado ("Qué soleco!” por "iQué solecismo!"), marcas de oralidad ("bueno, bueno") y frases coloquiales ("gané el vando"). Por otro lado, la interpretación de este poema como sátira a la retórica escolar nos parece menos factible, ya que Álvarez de Velasco no suele mezclar lo satírico y lo burlesco en la misma pieza.

El único poema profano que hemos encontrado entre los burlescos, antes de llegar a la parte final laudatoria, se titula "El derecho, y las leyes han de ser derechas en quanto el hombre lo puede ser". Una de sus estrofas dice lo siguiente:

Por esto, si yo entendí mal la ley por mi interés, construyéndola al rebés, yel por ley vengo a formar: 
pues si al rebés llega a estar,

ya es Derecho contra-hecho,

que por esto el Derecho es derecho (p. 401).

En este caso, no existe relación alguna con el tiempo de la fiesta y, por lo tanto, con sus códigos estéticos asociados. Es cierto que existen recursos literarios, como el calambur ("por ley"), comunes a esta muestra y a los villancicos comentados. Se trata, creemos, de un fenómeno de convergencia en torno a ciertos elementos superficiales, lo que no anula su diferenciación estética. En este último poema, el marco referencial ya no es la cultura popular, sino que encontramos una afiliación al conceptismo, corriente de poesía culta en que se adscribe a Francisco de Quevedo. Para entender cabalmente esta diferencia, debemos iniciar el estudio de la "esfera intermedia".

Rhythmica laudatoria, o la esfera intermedia

En el mundo actual, la risa literaria ya no se asocia al tiempo de la fiesta. En efecto, podemos encontrarla en todo tipo de obras en verso y en prosa, así como en numerosas manifestaciones culturales, como el cine o la publicidad. Esto se debe a que en la modernidad se produce un diálogo entre los ámbitos de la seriedad y la risa, hasta el punto de que es muy difícil establecer distinciones. La poesía, por ejemplo, puede tratar un tema elevado con expresiones coloquiales, lo que en otra época hubiera sido una falta de "decoro".

En la Nueva Granada del siglo XVII, existían claras barreras entre las distintas estéticas. Hemos visto cómo el poeta Álvarez de Velasco es un caso extremo por su diferenciación entre los poemas insertos en la alta cultura, siempre seria; y aquellos que utilizan los recursos de la cultura popular en obras asociadas al tiempo de la fiesta, como los villancicos navideños. Sin embargo, en su obra también se aprecia, aunque en menor medida, un tercer camino que Beltrán Almería (2016) denomina "esfera intermedia". Se trata de "un momento histórico en el que la literatura festiva se emancipa de los rituales de la fiesta para formar parte de la cultura de la escritura" (p. 30). Este momento, que Beltrán sitúa en el siglo XVI para la Europa Occidental (y del que Francisco de Quevedo es uno de sus principales representantes), no se manifiesta de forma homogénea en la literatura hispánica de la Colonia, quizás por el énfasis con que la ciudad letrada trataba de establecer la exclusividad y dignidad de la "alta cultura" (recordemos la prohibición del género "intermedio" por excelencia, la novela).
En este contexto, el estudio de la sección laudatoria de la Rhythmica, resulta de gran interés. Se trata de un conjunto de poemas en los que el sujeto poético de Álvarez de Velasco muestra su profunda admiración, e incluso amor platónico, a Sor Juana Inés de la Cruz. El tono con que lo hace diferencia estos poemas de los anteriores. A modo de ejemplo, reproducimos el inicio de las "Endechas endecasílabas":

Paysanita querida, No te piques, ni te alteres, Que tambien son Paysanos Los Angeles divinos, y los Duendes.

Yo soy este, que trasgo Amante inquieto, siempre En tu celda invisible, Haciendo ruido estoy con tus papeles (p. 562).

El vocativo del primer verso marca el tono familiar con que Álvarez de Velasco decide dirigirse a la poeta mexicana. Por supuesto, como ha destacado la crítica, también es una muestra de conciencia regional, sin que esto suponga un deseo de distanciamiento respecto a la metrópoli (de hecho, se celebra el prestigio que sor Juana irradia a "Nuestros invictos Reyes", p. 545). Lo que sigue, por las expresiones coloquiales ("no te piques"), y por el imaginario empleado (los duendes o trasgos) hace pensar en la cultura popular. 
También existen convergencias en otros recursos, como el ya citado calambur, que en las cartas laudatorias encontramos en versos como "A Soror Nise dicha, / (Que aun sin nombrarla, siempre Nise es dicha)", en los que se explota la homonimia entre el participio del verbo decir y el sustantivo sinónimo de felicidad. El juego burlesco con el lenguaje es constante en estas epístolas en verso:

Es del Parnaso Emperatriz, y Apola

(Mas si algun culto lo de Apola acusa

Sepa, que tambien ay un Moro Musa) (p. 529).

Aquí, la feminización de Apolo, director de las Musas en la mitología griega, da pie a un nuevo calambur con la homonimia de "Musa", término referido a las inspiradoras del arte en el mundo grecolatino y también al gobernador musulmán que dirigió la conquista de España en el año 711. No existe, según vemos, una intención satírica, puesto que la defensa de la feminización de Apolo se resuelve en un simple juego de palabras, y no en una suerte de reivindicación feminista.

Con todo, los poemas laudatorios se diferencian profundamente de los villancicos que estudiamos en el epígrafe anterior, por tres motivos principales. En primer lugar, en estos poemas Álvarez de Velasco emplea la forma epistolar, lo que se asocia de forma clara al mundo de la escritura, a diferencia de los villancicos, que podían ser cantados. En segundo lugar, en estos poemas epistolares se introducen referentes concretos, cotidianos e individualizados (por ejemplo, "tu celda", refiriéndose a la habitación de la monja), en contraposición con el imaginario colectivo y tradicional de los villancicos (por ejemplo, el Portal de Belén). Por último, y quizás más significativo, los villancicos se sitúan en el tiempo cíclico de la fiesta, mientras que los poemas laudatorios marcan de forma explícita su ubicación en el tiempo histórico y lineal: "Lunes diez y nueve del corriente" (p. 569).

La Rhythmica laudatoria, por lo tanto, pertenece a un ámbito estético diferente. Un ámbito que se acerca a la estética moderna, motivo por el cual, según creemos, la crítica ha mostrado su preferencia hacia estos poemas, en detrimento de la poesía artificiosa (que aquí no hemos estudiado), la sátira moral sin risa, $\mathrm{y}$ lo burlesco asociado a géneros populares como los villancicos. Citemos como muestra al primer historiador de la poesía neogranadina, Vergara y Vergara (1958), quien afirma que "si [Álvarez de Velasco] hubiera cultivado más el género al que pertenecen las endechas reales, probablemente habría sido más conocido y ocuparía un lugar entre los buenos poetas de su época" (p. 188). En la actualidad, la crítica se muestra más preocupada por su propio lugar de enunciación, por lo cual preferimos omitir juicios sobre qué poemas son "mejores", y nos limitamos a señalar su acercamiento a la estética moderna (sin que este acercamiento suponga un juicio de valor, sino una constatación histórica).

\section{Conclusión}

Hasta el momento, la crítica no ha intentado comprender desde una perspectiva amplia las distintas manifestaciones de la risa en la poesía colonial. En parte, esto se debe a los prejuicios a favor de lo serio que todavía persisten entre los académicos. De esta manera, la presencia de la risa ha sido consignada en frases superficiales que la entienden como un elemento pintoresco y secundario. Análogamente, el tradicional estudio de los autores en torno a periodos y movimientos literarios, usados como compartimentos estancos, ha generado una cortedad de miras que impide un análisis de más amplio calado. En el caso de Álvarez de Velasco, gran parte de la crítica se ha dedicado a cuantificar el contenido barroco o clasicista de sus versos. El otro gran interés de los estudiosos, ha sido identificar en el autor los supuestos rasgos de un criollismo proto-nacional, confundiéndose las intenciones del autor con el deseo de sus intérpretes. 
El presente artículo propone un novedoso enfoque, basado en la obra teórica de Beltrán Almería, quien estudia, en una perspectiva amplia, las diversas manifestaciones de la estética de la risa. La poesía de Álvarez de Velasco supone, en este contexto, una oportunidad muy interesante para analizar las exclusiones, pervivencias y novedades en un periodo de encrucijada. Las complejidades, y no pocas contradicciones, de la élite criolla en el territorio colonial aumentan el interés de esta obra literaria.

Precisamente, una de las principales preocupaciones de la ciudad letrada en este momento parece ser la exclusión de las pasiones irracionales, asociadas a los pueblos indígenas, por lo que se radicaliza la marginación de la risa. Al mismo tiempo, la cultura popular muestra su pervivencia incluso en la obra de estos letrados, sobre todo en poemas destinados a festividades religiosas. Es importante notar que, al menos en el caso de Álvarez de Velasco, nos estamos refiriendo a la cultura popular hispana, lo que supone una nueva exclusión de los modelos autóctonos. Por último, en las cartas laudatorias a Sor Juana se abre un espacio, diferenciado de los anteriores, en el que la seriedad y la risa interactúan de una forma que se acerca a la estética moderna.
La principal limitación de este estudio es, al mismo tiempo, una apertura hacia nuevas vías de investigación. Aunque la poesía de Álvarez de Velasco nos parezca muy significativa, el presente artículo se limita a un solo autor neogranadino, por lo que resulta necesario poner estos hallazgos en relación con otras obras de la Colonia. Por ejemplo, la propia Sor Juana Inés de la Cruz, cuyo empleo de la risa ha sido estudiado por la crítica, pero nunca desde esta perspectiva. También, sería interesante comparar los resultados hallados para la poesía colonial con un análisis más detallado de la literatura metropolitana, con el objetivo de explicitar las peculiaridades de cada ámbito cultural.

\section{Referencias}

Álvarez de Velasco y Zorrilla, F. (1989). Rhythmica sacra, moral y laudatoria. Bogotá: Instituto Caro y Cuervo.

Arango Ferrer, J. (1965). Raíz y desarrollo de la literatura colombiana. Bogotá: Ediciones Lerner.

Arellano, I. (2006). Las máscaras de Demócrito: en torno a la risa en el Siglo de Oro. En I. Arellano y V. Roncero (Eds.), Demócrito áureo. Los códigos de la risa en el Siglo de Oro (pp. 329-359). Sevilla: Renacimiento.

Bechara, Z. (1997). Literatura hispanoamericana colonial: primeros siglos de poesía colombiana. Frankfurt: Peter Lang.

Beltrán Almería, L. (2002). La imaginación literaria: la seriedad y la risa en la literatura occidental. Barcelona: Montesinos. 
Beltrán Almería, L. (2016). Estética de la risa. México: Ficticia.

Bernal, R. (2015). Mestizaje y criollismo en la literatura de la Nueva España del siglo XVI. México: Fondo de Cultura Económica.

Gaitán Bayona, J.L. (2015). Una risa en el desierto: el arte de la resurrección y sermones y prédicas del Cristo de Elqui. La Palabra, 27, 159-170.

Gómez Restrepo, A. (1953). Historia de la literatura colombiana (Vol. II). Bogotá: Ediciones de la Revista Bolívar.

Jurado Valencia, F. (2000). Historia de un poeta opacado por la historia: Francisco Alvarez, el enamorado de Sor Juana. Literatura: teoría, historia, critica, 2, 21-46.

Mazzotti, J. A. (1996). La heterogeneidad colonial peruana y la construcción del discurso criollo en el siglo XVII. En Asedios a la heterogeneidad cultural. Libro de homenaje a Antonio Cornejo Polar (pp. 173-196). Lima: Asociación Internacional de Peruanistas.

Menéndez Pelayo, M. (1952). Historia de la poesía hispanoamericana (Colombia). En Literatura colombiana. Estudios críticos (pp. 251-344). Bogotá: Biblioteca de Autores Colombianos.

Moraña, M. (1989). Para una relectura del barroco hispanoamericano: problemas críticos e historiográficos. Revista de crítica literaria latinoamericana, 15(29), 219-231.

Orjuela, H. H. (1992). Historia de la literatura colombiana. Literatura colonial (Vol. III). Bogotá: Kelly.

Otero Muñoz, G. (1945). Resumen de historia de la literatura colombiana. Bogotá: Librería Voluntad.

Pascual Buxó, J. (1999). El poeta colombiano enamorado de sor Juana. Bogotá: Universidad Nacional de Colombia.

Porras Collantes, E. (2000). Otras noticias -y las últimas- sobre la prosaica vida de Don Francisco Álvarez de Velasco y Zorrilla. Literatura: teoría, historia, critica, 2, 11-20.

Tello, J. (1989). Estudio Preliminar. En Álvarez de Velasco y Zorrilla, F. Rhythmica sacra, moral y laudatoria (XIX - XLV). Bogotá: Instituto Caro y Cuervo.

Torres Quintero, R. (1989). Presentación. En Álvarez de Velasco y Zorrilla, F. Rhythmica sacra, moral y laudatoria (XV - XVIII). Bogotá: Instituto Caro y Cuervo.

Vergara y Vergara, J. M. (1958). Historia de la literatura en Nueva Granada. Desde la conquista hasta la independencia (1538 - 1820). (Vol. I). Bogotá: Biblioteca de la Presidencia de Colombia. 